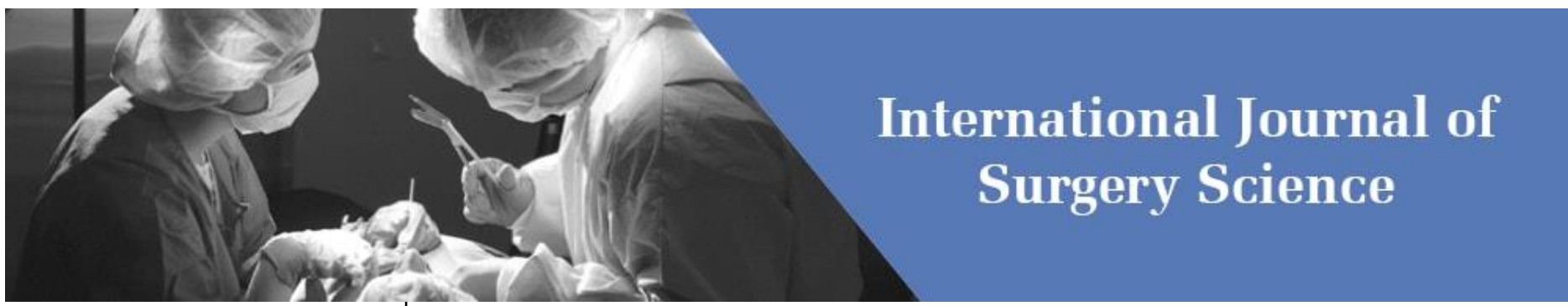

E-ISSN: 2616-3470

P-ISSN: 2616-3462

(C) Surgery Science

www.surgeryscience.com

2020; 4(2): 207-210

Received: 01-02-2020

Accepted: 03-03-2020

Dr. Pradeep Kumar J

Department of General Surgery, PES Institute of Medical Sciences and Research, Kuppam, Andhra Pradesh, India
Corresponding Author: Dr. Pradeep Kumar J Department of General Surgery, PES Institute of Medical Sciences and Research, Kuppam, Andhra Pradesh, India

\section{Routine lateral sphincterotomy with open haemorrhoidectomy: Our experience of 100 cases}

\section{Pradeep Kumar J}

DOI: https://doi.org/10.33545/surgery.2020.v4.i2d.417

\section{Abstract}

Background: Various methods have been described for management of haemorrhoids. Milligan Morgan's open haemorrhoidectomy is considered gold standard and radically performed procedure. The most dreaded sequel and main concern to open haemorrhoidectomy is post-operative pain. Our aim was to assess the advantage and risks associated in performing lateral sphincterotomy routinely with open haemorrhoidectomy with special concern in relieving post op pain.

Methods and Material: This prospective study was conducted by selection of consecutive 100 cases presenting with symptoms and signs suggestive of grade 3 and grade 4 hemorrhoids. The data in the study was collected by the use of a pretested proforma to collect relevant information from individual patient in the post-operative period. The post-operative pain was assessed using visual analog scale (VAS) and analgesic usage. Early post-operative complications like urinary retention, post-operative bleeding, incontinence and late complications like anal stenosis were assessed and tabulated for a period of 6 months. Results: In our series, 55 patients experienced mild pain and 14 patients experienced severe pain after 24 hours. On $3^{\text {rd }}$ post-operative day only 5 patients and after 1 week none of the patients complained of severe pain. Half of the patients required average of 0-2 analgesics/day but 15 patients demanded for more than 4 analgesic doses to relieve the pain. In the early post-operative period 20 patients were catheterized. 89 patients had confined bleeding and 11 patients had moderate bleeding. temporary incontinence was observed in 11 patients. During 6 months follow up none of our patients had anal stenosis.

Conclusions: Post haemorrhoidectomy pain still remains as challenging complication for surgeons following open haemorrhoidectomy because of its frequency. Lateral internal sphincter my which relieves the sphincter spasm can be tried routinely to relieve pain without significantly contributing to morbidity.

Keywords: Haemorrhoidectomy, lateral sphincterotomy, post haemorrhoidectomy pain

\section{Introduction}

It has been stated that $50 \%$ of the population will experience symptomatic haemorrhoid disease at some point in their lives ${ }^{[1]}$. But the exact prevalence of symptomatic haemorrhoids is very difficult to establish. Treatment options for symptomatic haemorrhoids that present to the clinic or emergency room range from non-operative medical interventions and outpatient based procedures to surgery. The ideal surgical treatment for haemorrhoids can be defined as safe, painless, minimally invasive with minimal costs. Although various methods have been proposed none of them meet all these features. The most commonly used method for treating third-degree and fourth-degree enlarged haemorrhoid is still Milligan-Morgan's open haemorrhoidectomy procedure. Complications accompanying open haemorrhoidectomy are significant postoperative pain, bleeding, incontinence, protracted recovery time and anal stricture.

Postoperative pain has historically been universal with surgical haemorrhoidectomy. The exact cause of pain after haemorrhoidectomy has not yet defined. Anal canal is richly innervated tissue in the digestive system, so pain after haemorrhoidectomy is an expected postoperative outcome. Various factors believed to be responsible for the pain including incarceration of smooth muscle fibres and mucosa in the transfixed vascular pedicle, epithelial denudation of the anal canal, and spasm of the internal sphincter. Another reason for pain could be the development of linear wounds extending up to the anorectal ring, which appear similar to those of a chronic anal fissure. No recurrence and reduction of pain after haemorrhoidectomy is an important goal along with reduction in the length of hospital stay.

For reduction of pain from the open wound of haemorrhoidectomy, various kinds of medication, including metronidazole, glyceryl trinitrate $(0.2 \%)$, steroids, local anaesthetics (bupivacaine), 
Anti-inflammatory drugs, haemorrhoid creams, are being used with variable outcome. In the postoperative period increased anal tone in the area of sensitive anal skin is considered to be a major pain producing factor. Various clinical trials have been done to decrease the sphincter tone. Anal canal dilatation was described by Lord in $1989^{[2]}$, but incidence of uncontrolled damage to the internal sphincter fibers was high. Eisenhammer [3] was the first to propagate the idea that post haemorrhoidectomy pain is due to spasm of the internal sphincter and described that its division through one of the haemorrhoid wounds is certainly an effective way to lessen postoperative pain.

In addition to pain, common complications in the early postoperative period include bleeding, urinary retention and in the later postoperative period include recurrent haemorrhoids, anal stenosis, skin tags, late haemorrhage, constipation (often due to narcotic use), and fecal incontinence. Lateral internal sphincterotomy (LIS) is a widely used adjunct treatment following open haemorrhoidectomy. Some researchers have reported limited ability of LIS to relieve postoperative pain and increased incidence of other complications, such as bleeding and fecal incontinence. Hence our study aims to assess the effectiveness of routine lateral sphincterotomy in relieving postoperative haemorrhoidectomy pain and any increase in morbidity with additional procedure.

\section{Subjects and Methods}

This study was conducted in single institution in 100 consecutive patients with grade 3 and grade 4 symptomatic haemorrhoidal diseases. Patients who satisfy the inclusion criteria were taken up for surgery after taking history, meticulous physical examination such as per rectal and anal speculum examination [proctoscopy] and appropriate serological investigations. All patients above 40 years underwent full colonoscopy before the operation to exclude any colonic pathology.

\section{Selection Criteria}

1. Patients aged between 18 to $70 \mathrm{yrs}$

2. Patients with grade 3 and grade 4 symptomatic haemorrhoids.

\section{Exclusion criteria}

1. Patients with complicated haemorrhoids like thrombosed piles, infected piles.

2. Patients with recurrent haemorrhoidal disease.

3. Patients had undergone perianal or per rectal procedures for any other diseases.

4. Patients who had received any other modality other than conservative treatment for existing disease.
5. Patients suffering from any other systemic or perianal disease along with haemorrhoids like crohn`s disease.

6. Patients having psychiatric illness.

7. Patients with known history of bleeding disorders.

Preoperative preparations, type of anaesthesia and the operative technique were same in all the patients. Patients with other medical illnesses were optimally treated and made normal before the surgery.

All the patients were subjected to elective open haemorrhoidectomy under spinal anaesthesia(S/A) and aseptic measures were taken. Vicryl 2-0 was used as suture material to secure pedicles. A $1 \mathrm{~cm}$ lateral incision made at left 3'0 clock position at the anal verge at haemorrhoidectomy wound site and Gentle dissection is done along the submucosa till the internal sphincter is reached. The lower free end of internal sphincter is hooked out using artery forceps and divided. Wound left open. Absolute haemostasis was maintained in the dissection bed in all the patients. Anal pack soaked in liquid paraffin and povidine iodine kept and dressing was done. The duration of surgery was noted.

All patients were given inj ceftriaxone $1 \mathrm{~g}$ at the time of induction of anesthesia and continued for 3 days in b.d, dosage. T. Metronidazole $400 \mathrm{mg}$ T.I.D. was added routinely for 3 days in all patients. On the 1st postoperative day, anal packing was removed after sitz bath with luke warm water. Patients were given a Sitz bath after passing stool each time. They were advised to apply ointment before passing stools and after each Sitz bath. All patients were kept on stool softeners \& sitz bath thrice a day for 2 weeks.

All the patients were given opioid analgesics (Inj. Tramadol $50 \mathrm{mg}$ i.m) at night of surgery. From the next day patients were given oral analgesics in the form of tablet Diclofenac Sodium 50g. Analgesic tablets were dispensed strictly on demand by patient. Our aim was to keep the post operative pain between 0 to 4 in visual analog score (VAS) and details of numbers of analgesic used were recorded against each patient. Pain was categorized mild if average of required analgesics to control pain were 0-2/day, moderate if analgesics required were 3-4/day and excruciating if more than 4 doses/day required.

Postoperative pain perception was measured using visual analog score (VAS). Score ranges from 0 to 10.A score of $>7$ is considered as severe pain, a score between 4-6 considered moderate and 1-3 as mild pain. Patients were instructed about how to record pain score in the VAS sheets provided and then discharged as per their convenience. The scores against each patient were recorded at the intervals of $24 \mathrm{hrs}, 3^{\text {rd }}$ post op day and after 1 week.

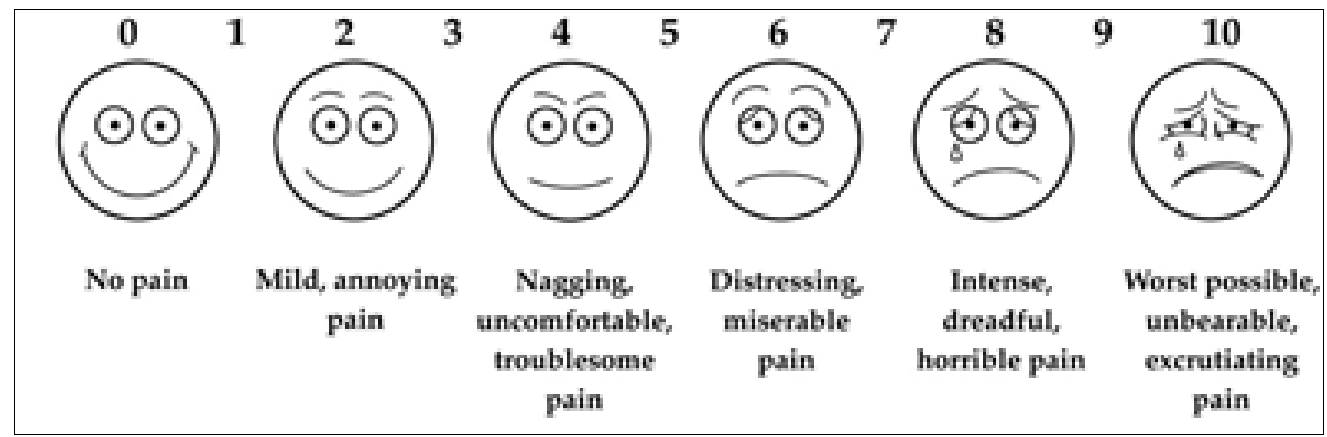

Fig 1: Visual analog scale (VAS). 
In the post-operative period, factors taken into account for study such as urinary retention, bleeding, post op pain status, difficulty in bowel continence were assessed thoroughly. The incidence of urinary retention following surgery was tabulated by considering the number of patients requiring catheterisation to relieve retention and not passed urine voluntarily or relieved by conservative measures.

We used Browning and Park's incontinence scale to measure the type and nature of incontinence. This method divides the patients into four groups: normal continence (i.e.continent for solids, liquid stools and flatus), continent for solid and liquid stools but not for flatus (gas incontinent patients), continent for solid stools only, usually presented with fecal leakage and complete incontinent patients. We have not evaluated the type of incontinence i.e. permanent or intermittent.

The amount of post operative bleeding was observed and assessed subjectively. It was grouped as Confined - the minimal loss which was bound to occur, Moderate - the loss of more than $20 \mathrm{ml}$ of blood or above normal permissible levels as observed by the patient and severe if the amount that was similar to the pre operative levels. All the patients were followed up for 6 months and any complications including anal stenosis during this period were assessed and tabulated.

\section{Results}

A total of 100 eligible patients were observed in our study. The lateral sphincterotomy procedure at the end of open haemorrhoidectomy took hardly few minutes with mean duration of surgery was $23.15 \mathrm{~min}$. As mentioned, assessment of pain was carried out with two variables; VAS and Number of analgesics administered. Severities of pain at different intervals are demonstrated in Table 1.

Table 1: Severity of pain at different intervals.

\begin{tabular}{|c|c|c|c|}
\hline Severity of pain & 24 hours & $\mathbf{3}^{\text {rd }}$ POD & 1 week \\
\hline Mild & 55 & 79 & 94 \\
\hline Moderate & 31 & 16 & 6 \\
\hline Severe & 14 & 5 & 0 \\
\hline
\end{tabular}

The severity of the pain was also evident by average number of analgesic dosages demanded by the patient. Half of the operated cases required 0 to 2 analgesics per day. 35 patients needed 3 to 4 doses/day whereas 15 patients required more than 4 doses/day to keep VAS score between 0 to 4 . The number of analgesics demanded by the patient decreased day by day as severity of pain also gradually decreased.

In the immediate post-operative period 20 patients were catheterised as they failed to pass urine voluntarily even after weaning of anaesthetic effect and conservative methods. As post-operative bleeding is one of the important complications following open haemorrhoidectomy, patients' data was collected and tabulated. Our patients were discharged according to their convenience and mean time required for them to return to their work was 20.16 days.

Patients were followed up by the same surgical team which performed the procedure and scheduled for visit at $1^{\text {st }}$ month, at $3^{\text {rd }}$ month and at $6^{\text {th }}$ month after discharge. 11 patients had various grades of incontinence in the immediate post op period. During follow up 6 patients improved at 1 month. By $3^{\text {rd }}$ and $6^{\text {th }}$ month all were relieved of incontinence and were comfortable. None of our patients suffered anal stenosis or required repeated anal dilatations.
Table 2: Incidence of post-operative complications

\begin{tabular}{|c|c|c|}
\hline Post op complication & \multicolumn{2}{|c|}{ Number of patients N=100 } \\
\hline \multirow{3}{*}{ Bleeding } & Confined & 89 \\
\cline { 2 - 3 } & Moderate & 11 \\
\cline { 2 - 3 } & Severe & 0 \\
\hline \multirow{2}{*}{ Urinary retention } & Yes & 20 \\
\cline { 2 - 3 } & No & 80 \\
\hline \multirow{3}{*}{ Incontinence } & Continent & 89 \\
\cline { 2 - 3 } & Gas defecation incontinence & 3 \\
\cline { 2 - 3 } & Liquid defecation incontinence & 4 \\
\cline { 2 - 3 } & Normal defecation incontinence & 4 \\
\hline
\end{tabular}

\section{Discussion}

Out of all the management options including injection sclerotherapy, infrared photocoagulation, and rubber band ligation, open haemorrhoidectomy is the gold standard operation against which the outcomes of the other treatment strategies are compared. However, postoperative pain remains a dreadful complication for some patients. Ascanelli concluded in a study that open haemorrhoidectomy was associated with higher postoperative pain scores ${ }^{[4]}$. A variety of surgical techniques and medical agents are available to the clinician in the treatment of post haemorrhoidectomy pain. But none of them proved to be superior. Several authors reported that significant reduction of post haemorrhoidectomy pain and associated complications can be achieved by adding internal sphincterotomy to haemorrhoidectomy.

The mechanism of action of lateral anal sphincterotomy on reducing the post operative pain following haemorrhoidectomy is still not yet confirmed but it has been shown to reduce the anal sphincteric tone causing the laxity of skin outside the perianal tissues leading to decrease in nerve stimulation and thereby decreasing the post operative pain. Di Bella and Estienne in 1990 suggested that internal sphincterotomy removed pain by reduction of sphincter tonicity ${ }^{[5]}$. In our study number of patients who suffered from severe post operative pain were very few. 14 patients had severe pain after 24 hours. Their severity of pain reduced in 48 hours. By the end of $3^{\text {rd }}$ post op day only 5 patients had experienced severe pain and only 15 patients were requiring more analgesic dosage to alleviate pain. In a study by Chauhan A. et al. it was revealed markedly reduced postoperative pain in patients who underwent haemorrhoidectomy with LIS.

Urinary retention is one of the most common complications following anorectal surgery with rates varying between 3 and $50 \%$ with most studies reporting a rate around $15 \%{ }^{[6]}$. In our study 20 patients developed urinary retention in post-operative period and required catheterization.

Lateral internal sphincterotomy at the end of open haemorrhoidectomy does not increase the operative time as procedure took hardly few minutes to perform using knife or cautery. Das et al. ${ }^{[7]}$ and Taha ${ }^{[8]}$ reported a shorter hospital stay for patients who underwent open haemorrhoidectomy with lateral sphincterotomy. However in our study the mean duration of hospital stay was 8.45 days and mean of return to work after surgery was 19.16 days.

Postoperative bleeding is a particularly important complication in treating hemorrhoids due to its frequency. For conventional haemorrhoidectomy (Milligan-Morgan and Ferguson) rates of clinically significant haemorrhage has been reported in the range of 0.3 to $6 \%$, with an average of around $2 \%{ }^{[9]}$. In our study, all patients experienced rectorrhagia. It was mainly confined in 
$89 \%$ of cases and those who had moderate bleeding were improved by $7^{\text {th }}$ post-operative day. None of our patients were suffered severe post-operative bleeding requiring any intervention or blood transfusion.

Literature has documented cases where sphincterotomy during open haemorrhoidectomy is accused as causing incontinence ${ }^{[7]}$. The incontinence grade ranges from $1.3 \%$ to $2.9 \%$. In DK Das's study with 50 patients, one patient developed fecal soiling (lasted for 2 weeks) and 2 patients developed temporary flatus incontinence ${ }^{[10]}$. In our study 8 patients had experienced incontinence which is of mild grade and temporary. All patients recovered well by the end of 6 months. In our follow up of patients we didn't encounter any anal stenosis which is considered as uncommon complication of haemorrhoidectomy.

Studies on measurement of anal sphincter pressure show that the basal anal pressure is significantly higher in patients with hemorrhoids and lateral internal sphincterotomy is more useful in young patients with higher anal pressure and its role in elderly patients and patients with normal basal anal pressure is doubtful. Anal manometry was not performed in our study due to logistic problems.Our study didn't reflect the incidence of true anal incontinence and Chronic anal pain after anorectal surgery which can be disabling for the patient and difficult to treat as the period of follow-up was rather short.

\section{Conclusion}

Lateral internal sphincterotomy is an easy simple procedure that can be carried out routinely with open haemorrhoidectomy without prolongation of duration of surgery. Most of the haemorrhoids patients will be having increased anal pressure and spasm is one of the main mechanisms in producing post haemorrhoidectomy pain. Hence lateral internal sphincterotomy which reduces the sphincter tone helps in alleviating the pain in significant number of cases. The chances of increase in complications by addition of lateral internal sphincterotomy are not so more than the main procedures i.e. open haemorrhoidectomy. Considering the advantages of lateral internal sphincterotomy the morbidity associated with additional procedure are acceptable.

\section{References}

1. Baker H. Hemorrhoids. In JL. Longe (Ed.) Gale encyclopedia of medicine. $3^{\text {rd }}$ ed.Gale, Detroit. 2006, 17661769.

2. Lord PH. Digital dilatation for hemorrhoids treatment. Int Surg. 1989; 74:144-145.

3. Eisehammer S. Internal anal sphincterotomy plus free dilatation versus anal stretch with special criticism of the anal stretch procedure for haemorrhoids: The recommended modern approach to haemorrhoids treatment. Dis Colon Rectum. 1974; 17:493-522.

4. Ascanelli S, Gregorio C, Tonini G, Baccarini M. Long stapled haemorrhoidectomy versus Milligan-Morgan procedure. ChirItal. 2005; 57(4):439-47.

5. Di Bella F, Estienne G. Indications for internal, medioposterior with anoplasty or lateral sphincterectomy during haemorrhoidectomy. Our experience [in Italian]. Minerva Chir. 1990; 45:361-363.

6. Toyonaga T, Matsushima M, Sogawa N. et al. Postoperative urinary retention after surgery for benign anorectal disease: potential risk factors and strategy for prevention. Int. J Colorectal Dis. 2006; 21(7):676-682.

7. Das DK, Choudhury UC, Lim ZS. Effectiveness of internal sphincterotomy in reducing post hemorrhoidal pain: A randomized comparative clinical study. International journal of collaborative research on internal medicine \& public health. 2013; 5(6):428-35.

8. Taha SA. Routine internal sphincterotomy with haemorrhoidectomy for third and fourth degree haemorrhoids greatly improves the outcome. IJGE. 2013; $1: 48-51$

9. Cintron J, Abcarian H. New York NY: Springer, Benign anorectal: haemorrhoids, 2007, 156-177.

10. Abramowitz L, Sobhani I, Benifla JL et al. Anal fissure and thrombosed external haemorrhoids before and after delivery. Dis Colon Rectum. 2002; 45:650-5. 